\title{
Fluctuation-Response Relation and modeling in systems with fast and slow dynamics
}

\author{
G. Lacorata ${ }^{1}$ and A. Vulpiani ${ }^{2}$ \\ ${ }^{1}$ Institute of Atmospheric and Climate Sciences, National Research Council, Str. Monteroni, 73100 Lecce, Italy \\ ${ }^{2}$ Department of Physics and INFN, University of Rome "La Sapienza", P.le A. Moro 2, 00185 Roma, Italy
}

Received: 27 June 2007 - Revised: 13 September 2007 - Accepted: 18 October 2007 - Published: 31 October 2007

\begin{abstract}
We show how a general formulation of the Fluctuation-Response Relation is able to describe in detail the connection between response properties to external perturbations and spontaneous fluctuations in systems with fast and slow variables. The method is tested by using the 360variable Lorenz-96 model, where slow and fast variables are coupled to one another with reciprocal feedback, and a simplified low dimensional system. In the Fluctuation-Response context, the influence of the fast dynamics on the slow dynamics relies in a non trivial behavior of a suitable quadratic response function. This has important consequences for the modeling of the slow dynamics in terms of a Langevin equation: beyond a certain intrinsic time interval even the optimal model can give just statistical prediction.
\end{abstract}

\section{Introduction}

One important aspect of climate dynamics is the study of the response to perturbations of the external forcings, or of the control parameters. In very general terms, let us consider the symbolic evolution equation:

$$
\frac{d \boldsymbol{X}}{d t}=\mathbf{Q}(\boldsymbol{X})
$$

where $\boldsymbol{X}$ is the state vector for the system, and $\mathbf{Q}(\boldsymbol{X})$ represents complicated dynamical processes. As far as climate modeling is concerned, one of the most interesting properties to study is the so-called Fluctuation-Response relation (FRR), i.e. the possibility, at least in principle, to understand the behavior of the system (1) under perturbations (e.g. a volcanic eruption, or a change of the $\mathrm{CO}_{2}$ concentration) in terms of the knowledge obtained from its past time history (Leith, 1975, 1978; Dymnikov and Gritsoun, 2001).

Correspondence to: G. Lacorata

(g.lacorata@isac.cnr.it)
The average effect on the variable $X_{i}(t)$ of an infinitesimal perturbation $\delta \mathbf{f}(t)$ in Eq. (1), i.e. $\mathbf{Q}(\boldsymbol{X}) \rightarrow \mathbf{Q}(\boldsymbol{X})+\delta \mathbf{f}(t)$, can be written in terms of the response matrix $\mathbf{R}_{i j}(t)$. If $\delta \mathbf{f}(t)=0$ for $t<0$ one has:

$\overline{\delta X_{i}(t)}=\sum_{j} \int_{0}^{t} R_{i j}\left(t-t^{\prime}\right) \delta f_{j}\left(t^{\prime}\right) d t^{\prime}$

where $\mathbf{R}_{i j}(t)$ is the average response of the variable $X_{i}$ at time $t$ with respect to a perturbation of $X_{j}$ at time 0 .

The basic point is, of course, how to express $\mathbf{R}_{i j}(t)$ in terms of correlation functions of the unperturbed system. The answer to this problem is the issue of the FluctuationResponse theory. This field has been initially developed in the context of equilibrium statistical mechanics of Hamiltonian systems; this generated some confusion and misleading ideas on its validity. As a matter of fact, it is possible to show that a generalized FRR holds under rather general hypotheses (Deker and Haake, 1975; Falcioni et al., 1990): the FRR is also valid in non Hamiltonian systems. It is interesting to note that, although stochastic and deterministic systems, from a conceptual (and technical) point of view, are somehow rather different, the same FRR holds in both cases, see Appendix A. For this reason, in the following, we will not separate the two cases. In addition, a FRR holds also for not infinitesimal perturbation (Boffetta et al., 2003). From many aspects, the FRR issues in climate systems are rather similar to those in fluids dynamics: we have to deal with non Hamiltonian and non linear systems whose invariant measure is non Gaussian (Kraichnan, 2000). On the other hand, it is obviously impossible to model climate dynamics with equations obtained from first principles, so typically it is necessary to work with simple raw models or just to deal with experimental signals (Ditlevsen, 1999; Marwan et al., 2003). In addition, in climate problems (and more in general in Geophysics) the study of infinitesimal perturbation is rather academic, while a much more interesting question is the relaxation of large perturbations in the system due to

Published by Copernicus Publications on behalf of the European Geosciences Union and the American Geophysical Union. 
fast changes of the parameters. Numerical simulations show that, in systems with one single time scale (e.g. low dimensional chaotic model as the Lorenz one), the amplitude of the perturbation is not so important, (see Appendix A, and Boffetta et al., 2003). On the contrary, in the case of different characteristic times, the amplitude of the perturbation can play a major role in determining the response, because different amplitudes may affect features with different time properties (Boffetta et al., 2003). Starting from the seminal works of Leith $(1975,1978)$, who proposed the use of FRR for the response of the climatic system to changes in the external forcing, many authors tried to apply this relation to different geophysical problems, ranging from simplified models (Bell, 1980), to general circulation models (North et al., 1999; Cionni et al., 2004) and to the covariance of satellite radiance spectra (Haskins et al., 1999). For recent works on the application of the FRR to the sensitivity problem and the predictability see Gritsoun and Dymnikov (1999), Gritsoun (2001), Gritsoun et al. (2002), Dymnikov and Gritsoun (2005), Dymnikov (2004), Abramov and Majda (2007), and Gritsoun and Branstator (2007). In most works, the FRR has been invoked in its Gaussian version, see below, which has been used as a kind of approximation, often without a precise idea of its limits of applicability. In principle, according to Lorenz (1996), one has to consider two kinds of sensitivity: to the initial conditions (first kind) and to the parameters (e.g. external forcing) of the system (second kind). On the other hand, if one considers just infinitesimal perturbations, it is possible to describe the second kind problem in terms of the first one. Unfortunately, this is not true for non infinitesimal perturbations.

In this paper we study, in the FRR framework, systems with more than one characteristic time. In Sect. 2 we recall the theoretical basis of the FRR issue. In Sect. 3 we describe the analysis we have performed on two dynamical systems. The first one, is a model introduced by Lorenz (1996), which contains some of the relevant features of climate systems, i.e. the presence of fast and slow variables (see Fraedrich, 2003 , for a discussion about short and long-term properties of complex multiscale systems like the atmosphere). We consider, at this regard, the problem of the parameterization of the fast variables via a suitable renormalization of the parameters appearing in the slow dynamics equations, and the addition of a random forcing. The second one is a very simplified system consisting, basically, of a slow variable which fluctuates around two states, coupled to fast chaotic variables. The specific structure of this system suggests a modeling of the slow variable in terms of a stochastic differential equation. We will see how, even in absence of a Gaussian statistics, the correlation functions of the slow (fast) variables have, at least, a qualitative resemblance with response functions to perturbations on the slow (fast) degrees of freedom. In addition, although the average response of a slow variable to perturbations of the fast components is zero, the influence of the fast dynamics on the slow dynamics cannot be ne- glected. This fact is well highlighted by a non trivial behavior of a suitable quadratic response function (Hohenberg and Shraiman, 1989). In the framework of the complexity in random dynamical systems, one has to deal with a similar behavior: the relevant "complexity" of the system is obtained by considering the divergence of nearby trajectories evolving with two different noise realizations (Paladin et al., 1995). This has important consequences for the modeling of the slow dynamics in terms of a Langevin equation: beyond a certain intrinsic time interval (determined by the shape of the quadratic response function) even the optimal model can give just statistical predictions (for general discussion about the skills and the limits of predictability of climatic models see Cane, 2003). The conclusions and the discussion of the results obtained in this work are contained in Sect. 4, while the Appendices are devoted to some technical aspects.

\section{Theoretical background on FRR}

For the sake of completeness we summarize here some basic results regarding the FRR (see Appendix A for technical details). Let us consider a dynamical system $\boldsymbol{X}(0) \rightarrow \boldsymbol{X}(t)=U^{t} \boldsymbol{X}(0)$ whose time evolution can even be not completely deterministic (e.g. stochastic differential equations), with states $\boldsymbol{X}$ belonging to a $\mathrm{N}$-dimensional vector space. We assume: a) the existence of an invariant probability distribution $\rho(\boldsymbol{X})$, for which some "absolute continuity" type conditions are required (see Appendix A); b) the mixing $^{1}$ character of the system (from which its ergodicity follows).

At time $t=0$ we introduce a perturbation $\delta \boldsymbol{X}(0)$ on the variable $\boldsymbol{X}(0)$. For the quantity $\overline{\delta X_{i}}(t)$, in the case of an infinitesimal perturbation $\delta \boldsymbol{X}(0)=\left(\delta X_{1}(0) \cdots \delta X_{N}(0)\right)$ one obtains:

$\overline{\delta X_{i}}(t)=\sum_{j} R_{i j}(t) \delta X_{j}(0)$

where the linear response functions (according to FRR) are

$R_{i j}(t)=-\left\langle\left. X_{i}(t) \frac{\partial \ln \rho(\boldsymbol{X})}{\partial X_{j}}\right|_{t=0}\right\rangle$.

In the following $\langle()\rangle$ indicates the average on the unperturbed system, while () indicates the mean value of perturbed quantities. The operating definition of $\mathbf{R}_{i j}(t)$ in numerical simulations is the following. We perturbe the variable $X_{j}$ at time $t=t_{0}$ with a small perturbation of amplitude $\delta X_{j}(0)$ and then evaluate the separation component $\delta X_{i}\left(t \mid t_{0}\right)$ between the two trajectories $\boldsymbol{X}(t)$ and $\boldsymbol{X}^{\prime}(t)$ which are integrated up to a prescribed time $t_{1}=t_{0}+\Delta t$. At time $t=t_{1}$, the variable $X_{j}$ of the reference trajectory is again perturbed with the same $\delta X_{j}(0)$,

\footnotetext{
${ }^{1} \mathrm{~A}$ dynamical system is mixing if, for $t \rightarrow \infty$, $\left\langle f\left(U^{t} \boldsymbol{X}\right) g(\boldsymbol{X})\right\rangle \rightarrow\langle f(\boldsymbol{X})\rangle\langle g(\boldsymbol{X})\rangle$, where the average is over the invariant probability distribution and $f$ and $g$ are $L_{2}$ functions.
} 
and a new sample $\delta \boldsymbol{X}\left(t \mid t_{1}\right)$ is computed and so forth. The procedure is repeated $M \gg 1$ times and the mean response is then given by:

$R_{i j}(\tau)=\frac{1}{M} \sum_{k=1}^{M} \frac{\delta X_{i}\left(t_{k}+\tau \mid t_{k}\right)}{\delta X_{j}(0)}$.

Usually, in non Hamiltonian systems, the shape of $\rho(\boldsymbol{X})$ is not known, therefore relation (4) does not give a very detailed information. On the other hand the above relation shows that, anyway, there exists a connection between the mean response function $\mathbf{R}_{i j}$ and some suitable correlation function, computed in the unperturbed systems.

In the case of multivariate Gaussian distribution, $\ln \rho(\boldsymbol{X})=-\frac{1}{2} \sum_{i, j} \alpha_{i j} X_{i} X_{j}+$ const. where $\left\{\alpha_{i j}\right\}$ is a positive symmetric matrix, the elements of the linear response matrix can be written in terms of the usual correlation functions, $C_{i k}=\left\langle X_{i}(t) X_{k}(0)\right\rangle /\left\langle X_{i} X_{k}\right\rangle$, as:

$R_{i j}(t)=\sum_{k} \alpha_{j k}\left\langle X_{i}(t) X_{k}(0)\right\rangle$.

One important nontrivial class of systems with a Gaussian invariant measure is the inviscid hydrodynamics ${ }^{2}$, where the Liouville theorem holds, and a quadratic invariant exists (Kraichnan, 1959; Kraichnan and Montgomery, 1980; Bohr et al., 1998). Sometimes in the applications, in absence of detailed information about the shape of $\rho$, formula (5) is assumed to hold to some extent. Numerical studies of simplified models which mimic the chaotic behavior of turbulent fluids show that, since that stationary probability distribution is not Gaussian, Eq. (5) does not hold. On the other hand, the correlation functions and the response functions have similar quantitative behavior. In particular, in fully developed turbulence, as one can expect on intuitive ground, one has that the times characterizing the responses approximate the characteristic correlation times (Biferale et al., 2002; Boffetta et al., 2003). This is in agreement with numerical investigation (Kraichnan, 1966) at moderate Reynolds number of the Direct Interaction Approximation equations, showing that, although $R_{i i}(t)$ is not exactly proportional to the autocorrelation function $C_{i i}(t)$, if one compares the correlation times $\tau_{C}\left(\boldsymbol{k}_{i}\right)$ (e.g. the time after which the correlation function becomes lower than 1/2) and the response time $\tau_{R}\left(\boldsymbol{k}_{i}\right)$ (e.g. the time after which the response function becomes lower than $1 / 2)$, the ratio $\tau_{C}\left(k_{a}\right) / \tau_{R}\left(k_{a}\right)$ remains constant through the inertial range. In the turbulence context, $X_{i}$ indicates the Fourier component of the velocity field corresponding to a wave vector $\boldsymbol{k}_{i}$.

We would like briefly to remark a subtle point. From a rather general argument (see Appendix B), one has that all

\footnotetext{
${ }^{2}$ There exist also inviscid hydrodynamic systems with non quadratic conservation laws, and, therefore, non Gaussian invariant measure. Such cases can have relevance in the statistical mechanics of fluids (Pasmanter, 1994).
}

the (typical) correlation functions, at large time delay, have to relax to zero with the same characteristic time, related to spectral properties of the operator $\hat{\mathbf{L}}$ which rules the time evolution of the probability density function $P(\boldsymbol{X}, t)$ :

$\frac{\partial}{\partial t} P(\boldsymbol{X}, t)=\hat{\mathbf{L}} P(\boldsymbol{X}, t)$.

Using this result in a blind way, one has the apparently paradoxical conclusion that, in any kind of systems, all the correlation functions, relative to degrees of freedom at different scales, relax to zero with the same characteristic time. On the contrary, in systems with many different characteristic times (e.g. fully developed turbulence), one expects a whole hierarchy of times distinguishing the behavior at different scales (Frisch, 1995). The paradox is, of course, only apparent since the above argument is valid just at very long times, i.e. much longer than the longest characteristic time, and therefore, in systems with fluctuations over many different time-scales, this is not very helpful.

\section{Response of fast and slow variables}

Systems with a large number of components and/or with many time scales, e.g. climate dynamics models, present clear practical difficulties if one wants to understand their behavior in detail. Even using modern supercomputers, it is not possible to simulate all the relevant scales of the climate dynamics, which involves processes with characteristic times ranging from days (atmosphere) to $10^{2}-10^{3}$ years (deep ocean and ice shields), see Majda et al. (2005) and Majda and Wang (2006).

For the sake of simplicity, we consider the case in which the state variables evolve over two very different time scales:

$$
\begin{aligned}
& \frac{d \boldsymbol{X}_{s}}{d t}=\mathbf{f}\left(\boldsymbol{X}_{s}, \boldsymbol{X}_{f}\right) \\
& \frac{d \boldsymbol{X}_{f}}{d t}=\frac{1}{\epsilon} \mathbf{g}\left(\boldsymbol{X}_{s}, \boldsymbol{X}_{f}\right)
\end{aligned}
$$

where $\boldsymbol{X}_{s}$ and $\boldsymbol{X}_{f}$ indicate the slow and fast state vectors, respectively, $\epsilon \ll 1$ is the ratio between fast and slow characteristic times, and both $\mathbf{f}$ and $\mathbf{g}$ are $O(1)$. A rather general issue is to understand the role of the fast variables in the slow dynamics. From the practical point of view, one basic question is to derive effective equations for the slow variables, e.g. the climatic observable, in which the effects of the fast variables, e.g. high frequency forcings, are taken into account by means of stochastic parameterization. Under rather general conditions (Givon et al., 2004), one has the result that, in the limit of small $\epsilon$, the slow dynamics is ruled by a Langevin equation with multiplicative noise:

$\frac{d \boldsymbol{X}_{s}}{d t}=\mathbf{f}_{\text {eff }}\left(\boldsymbol{X}_{s}\right)+\widehat{\sigma}\left(\boldsymbol{X}_{s}\right) \boldsymbol{\eta}$

where $\eta$ is a white-noise vector, i.e. its components are Gaussian processes such that $\left\langle\eta_{i}(t)\right\rangle=0$ and 


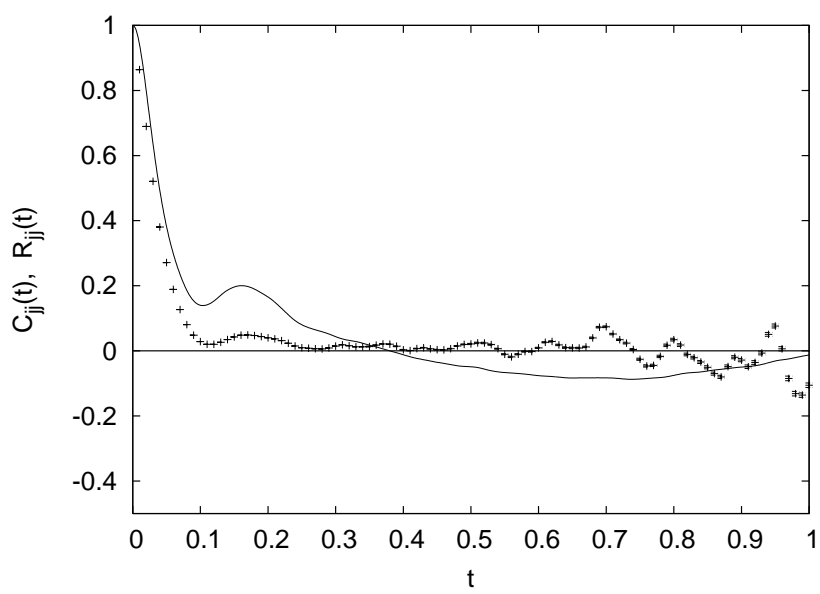

Fig. 1. Lorenz-96 model: autocorrelation $C_{j j}(t)$ (full line) and self-response $R_{j j}(t)(+)$ of the fast variable $y_{k, j}(t)(k=3, j=3)$. The statistical error bars on $R_{j j}(t)$ are of the same size as the graphic symbols used in the plot.

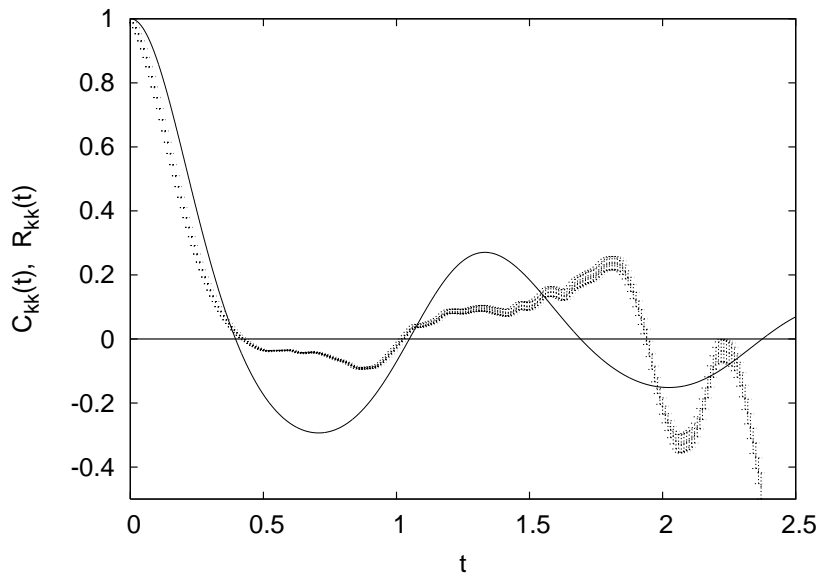

Fig. 2. Lorenz-96 model: autocorrelation $C_{k k}(t)$ (full line) and self-response $R_{k k}(t)$, with statistical error bars, of the slow variable $x_{k}(t)(k=3)$.

$\left\langle\eta_{i}(t) \eta_{j}\left(t^{\prime}\right)\right\rangle=\delta_{i j} \delta\left(t-t^{\prime}\right) . \quad$ Although there exist general mathematical results (Givon et al., 2004) on the possibility to derive Eq. (9) from Eqs. (7) and (8), in practice one has to invoke (rather crude) approximations based on physical intuition to determine the shape of $\mathbf{f}_{\text {eff }}$ and $\widehat{\sigma}$ (Mazzino et al., 2005). At this regard, see also the contribution to the volume by Imkeller and von Storch (2001) about stochastic climate models. For a more rigorous approach in some climate problems see Majda et al. $(1999,2001)$ and Majda and Franzke (2006).

In the following, we analyse and discuss two models which, in spite of their apparent simplicity, contain the basic features, and the same difficulties, of the general multi-

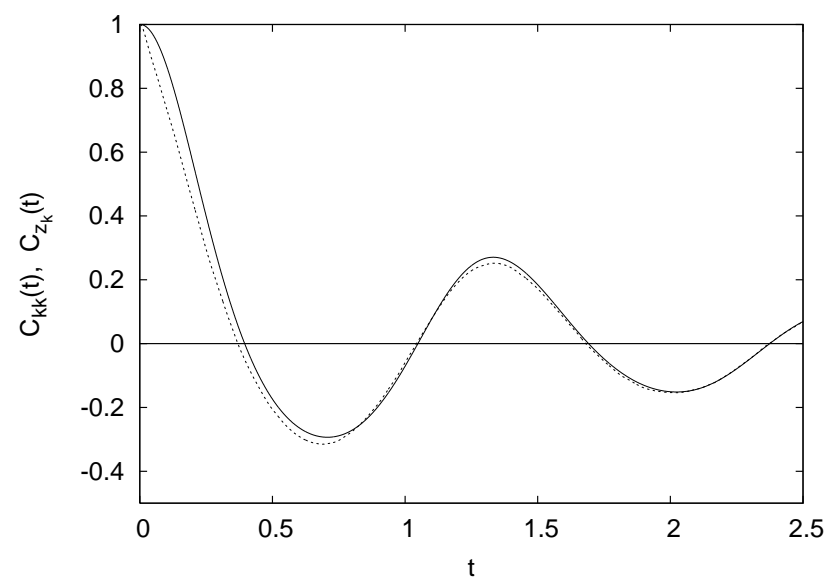

Fig. 3. Lorenz-96 model: autocorrelation $C_{z_{k}}(t)$ (dashed line) of the cumulative variable $z_{k}(t)$ compared to the autocorrelation $C_{k k}(t)$ of $x_{k}(t)$ (full line).

scale approach: the Lorenz-96 model (Lorenz, 1996) and a double-well potential with deterministic chaotic forcing.

\subsection{The Lorenz-96 model}

First, let us consider the Lorenz-96 system (Lorenz, 1996), introduced as a simplified model for the atmospheric circulation. Define the set $\left\{x_{k}(t)\right\}$, for $k=1, \ldots, N_{k}$, and $\left\{y_{k, j}(t)\right\}$, for $j=1, \ldots, N_{j}$, as the slow large-scale variables and the fast small-scale variables, respectively (being $N_{k}=36$ and $\left.N_{j}=10\right)$. Roughly speaking, the $\left\{x_{k}\right\}$ 's represent the synoptic scales while the $\left\{y_{k, j}\right\}$ 's represent the convective scales. The forced dissipative equations of motion are:

$\frac{d x_{k}}{d t}=-x_{k-1}\left(x_{k-2}-x_{k+1}\right)-v x_{k}+F+c_{1} \sum_{j=1}^{N_{j}} y_{k, j}$

$\frac{d y_{k, j}}{d t}=-c b y_{k, j+1}\left(y_{k, j+2}-y_{k, j-1}\right)-c v y_{k, j}+c_{1} x_{k}$

where: $F=10$ is the forcing term, $\nu=1$ is the linear damping coefficient, $c=10$ is the ratio between slow and fast characteristic times, $b=10$ is the relative amplitude between large scale and small scale variables, and $c_{1}=c / b=1$ is the coupling constant that determines the amount of reciprocal feedback.

Let us consider, first, the response properties of fast and slow variables, see Figs. 1 and 2.

In Fig. 1, the autocorrelation $C_{j j}(t)$ and self-response $R_{j j}(t)$ refer to the fast variable $y_{k, j}(t)$, with fixed $k$ and $j$. It is well evident how, even in absence of a precise agreement between autocorrelations and self-response functions (due to the non Gaussian character of the system), one has that the correlation of the slow (fast) variables have at least a 


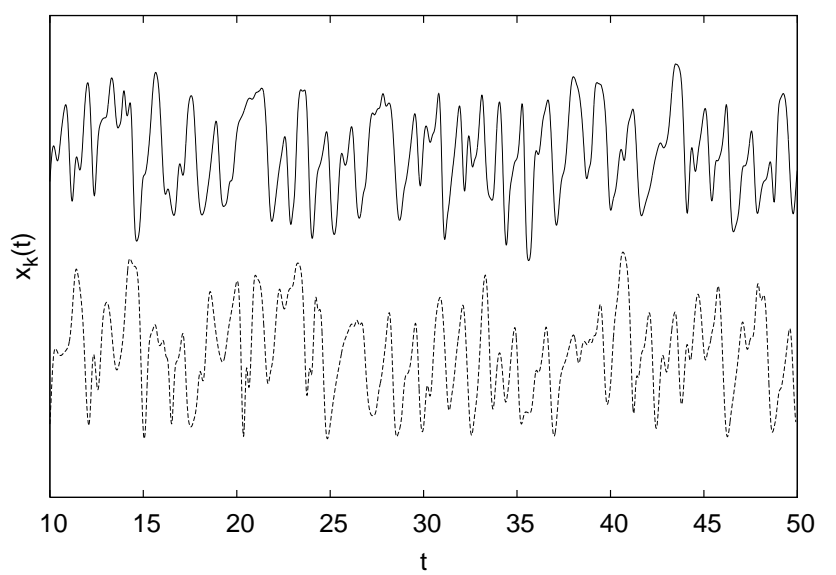

Fig. 4. Lorenz-96 model: time signal sample of the slow variable $x_{k}(t)(k=3)$ for the deterministic model (full line) and for the stochastic model (dashed line). For clarity, the two signals have been shifted from each other along the vertical axis.

qualitative resemblance with the response of the slow (fast) variables themselves.

The structure of the Lorenz-96 model includes a rather natural set of quantities that suggests how to parameterize the effects of the fast variables on the slow variables, for each $k$. Let us indicate with $z_{k}=\sum_{j=1}^{N_{j}} y_{k, j}$ the term containing all the $N_{j}$ fast terms in the equations for the $N_{k}$ slow modes. In the following, we will see that, replacing the deterministic terms $\left\{z_{k}\right\}$ 's in the equations for the $\left\{x_{k}\right\}$ 's with suitable stochastic processes, one obtains an effective model able to reproduce the main statistical features of the slow components of the original system.

It's worth-noting, from Fig. 3, that $C_{k k}(t)$ and $C_{z_{k}}(t)$, the autocorrelation of the cumulative variable $z_{k}(t)$, are rather close to each other. This suggests that $z_{k}(t)$ must be correlated to $x_{k}(t)$, in other words, the cumulative effects of the $N_{j}$ fast variables $y_{k, j}(t)$ on $x_{k}(t)$ are equivalent to an effective slow term, proportional to $x_{k}(t)$.

We look, therefore, for a conditional white noise parameterization that takes into account this important information given by the structure of the Lorenz-96 model equations. Let us write the effective equations for the slow modes as

$\frac{d x_{k}}{d t}=-x_{k-1}\left(x_{k-2}-x_{k+1}\right)-\left(v+v^{\prime}\right) x_{k}+\left(F+F^{\prime}\right)+c_{2} \cdot \eta_{k}$

where $\eta_{k}$ are uncorrelated and normalized white-noise terms. Some authors, Majda et al. (1999, 2001) and Majda and Franzke (2006), using multiscale methods, have obtained effective Langevin equations for the slow variables of systems having the same structure as the Lorenz-96 model.

Basically we can say that, in the effective model for the slow variables, one parameterizes the effects of the fast variables with a suitable renormalization of the forcing, $F \rightarrow F+F^{\prime}$, of the viscosity, $v \rightarrow v+v^{\prime}$, and the addition of a

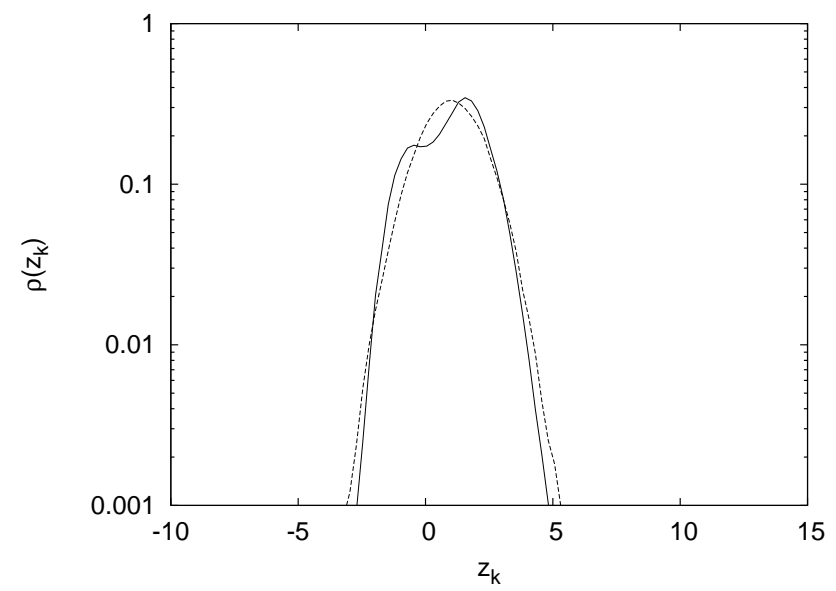

Fig. 5. Lorenz-96 model: PDFs of the cumulative variable $z_{k}$ $(k=3)$, see definition in the text for the two cases, for the deterministic model (full line) and the stochastic model (dashed line).

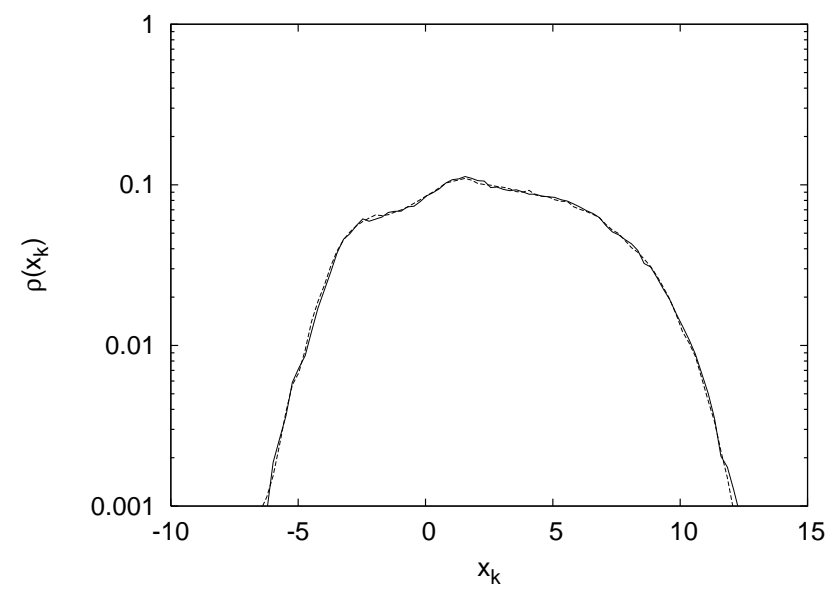

Fig. 6. Lorenz-96 model: PDFs of the slow variable $x_{k}(k=3)$ for the deterministic model (full line) and the stochastic model (dashed line).

random term. In other words, we replace the $z_{k}=\sum_{j=1}^{N_{j}} y_{k, j}$ terms in Eq. (10) with stochastic processes $\widetilde{z_{k}}$ depending on the slow variables $x_{k}$ :

$\frac{d x_{k}}{d t}=-x_{k-1}\left(x_{k-2}-x_{k+1}\right)-v x_{k}+F+\widetilde{c_{1}} \widetilde{z_{k}}$

where

$\widetilde{z_{k}}=\frac{1}{\widetilde{c_{1}}}\left(-v^{\prime} x_{k}+F^{\prime}+c_{2} \eta_{k}\right)$

with $\widetilde{c_{1}}$ is a new coupling constant. We notice that Eq. (13) has the same form of Eq. (10). With a proper choice of $v^{\prime}$, $F^{\prime}$ and $c_{2}$ in Eq. (12), $v^{\prime}=-0.3, F^{\prime}=0.25, c_{2}=0.3$, which implies $\widetilde{c_{1}}=0.25$ in Eq. (13), one can reproduce the statistics 


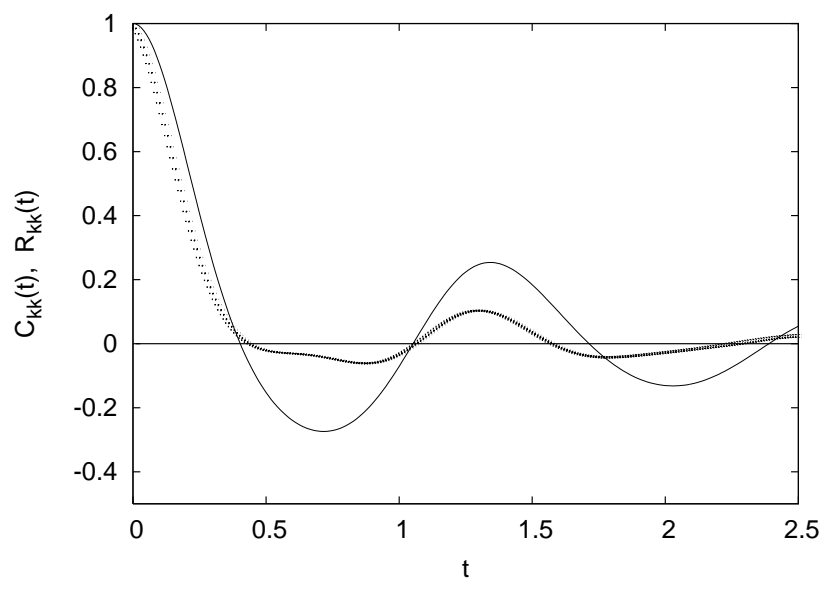

Fig. 7. Lorenz-96 model: autocorrelation $C_{k k}(t)$ (full line) and self-response $R_{k k}(t)$, with statistical error bars, of the slow variable $x_{k}(t)$ for the stochastic model.

of $x_{k}$ and $z_{k}$ to a very good extent, see at this regard Figs. 4, 5 and 6 . Of course the above described parameterization of the fast variables is inspired to the general "philosophy" of the Large-Eddy Simulation of turbulent geophysical flows at high Reynolds numbers (Moeng, 1984; Moeng and Sullivan, 1994; Sullivan et al., 1994).

The FR properties of the stochastic Lorenz-96 slow variables are reported in Fig. 7.

Let us come back to the response problem. Of course the mean response of a slow variable to a perturbation on a fast variable is zero. However, this does not mean that the effect of the fast variables on the slow dynamics is not statistically relevant. Let us introduce the quadratic response of $x_{k}(t)$ with respect to an infinitesimal perturbation on $y_{k, j}(0)$, for fixed $k$ and $j$ :

$R_{k j}^{(q)}(t)=\frac{\left[\overline{\delta x_{k}(t)^{2}}\right]^{1 / 2}}{\delta y_{k, j}(0)}$

Considered that in all simulations the initial impulsive perturbations on the $y_{k, j}$ is kept constant, $\delta y_{k, j}(0)=\Delta$, with $\Delta \ll\left\langle y_{k, j}^{2}\right\rangle^{1 / 2}$, it is convenient to take the average of Eq. (15) over all $j$ 's, at a fixed $k$, and introduce the quantity:

$R_{s f}^{(q)}(t)=\frac{\Delta}{N_{j}} \sum_{j=1}^{N_{j}} R_{k j}^{(q)}(t)$

where with $s$ and $f$ we label the slow and fast variables, respectively. In the case of the Lorenz-96 system, all the $y_{k, j}$ variables, at fixed $k$, are statistically equivalent, and have identical coupling with $x_{k}$, so that $R_{s f}^{(q)}(t) / \Delta$ coincides with $R_{k j}^{(q)}(t)$. We report in Fig. 8 the behavior of $R_{s f}^{(q)}(t)$, for both Eqs. (10) and (13). As regards to the stochastic model, the analogous of Eq. (16) is defined as follows. One studies the

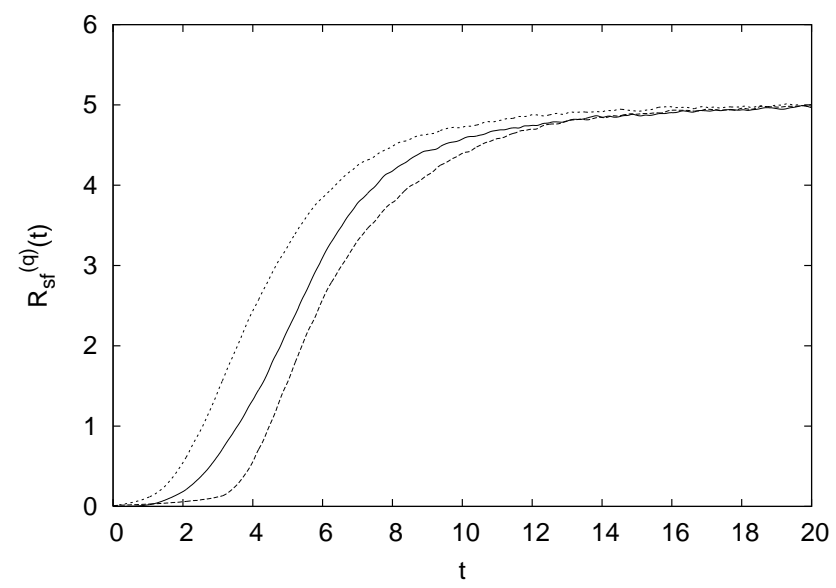

Fig. 8. Lorenz-96 model: quadratic cross-response function $R_{s f}^{(q)}(t)$ for the deterministic model (full line), for the stochastic model when the slow variables evolve with the same noise realization for all components except one (dashed line), and when the slow variables evolve with a different noise realization for every component (dotted line).

evolution of $\delta x_{k}(t)$ as difference of two trajectories obtained with two different realizations of the $\left\{\eta_{k}\right\}$ 's. It is worth stressing that the behavior of $\delta x_{k}(t)$ under two noise realizations can be very different from the behavior of $\delta x_{k}(t)$ under the same noise realization (see Appendix C). This aspect will be considered again in the next section.

\subsection{A simplified model}

In order to grasp the essence of systems with fast and slow variables, we discuss now a toy climate model in which the "climatic" variable fluctuates between two states. Consider a four dimensional state vector $\boldsymbol{q}=\left(q_{0}, q_{1}, q_{2}, q_{3}\right)$ whose evolution is given by:

$\frac{d q_{0}}{d t}=2 \sqrt{H} q_{0}-q_{0}^{3}+c q_{1}$

$\frac{d q_{1}}{d t}=\frac{1}{\widetilde{\epsilon}}\left[-\sigma_{L}\left(q_{1}-q_{2}\right)\right]$

$\frac{d q_{2}}{d t}=\frac{1}{\widetilde{\epsilon}}\left[-q_{1} q_{3}+r_{L} q_{1}-q_{2}\right]$

$\frac{d q_{3}}{d t}=\frac{1}{\widetilde{\epsilon}}\left[q_{1} q_{2}-b_{L} q_{3}\right]$

This four equation system will be named the deterministic DW model. The subsystem formed by Eqs. (18), (19) and (20) is nothing but the well-known Lorenz-63 model (Lorenz 1963), in which the constant $\widetilde{\epsilon}$ has the function of rescaling the characteristic time. In absence of coupling $(c=0)$ between $q_{0}$ and $q_{1}$, the unforced motion equation holds for the 


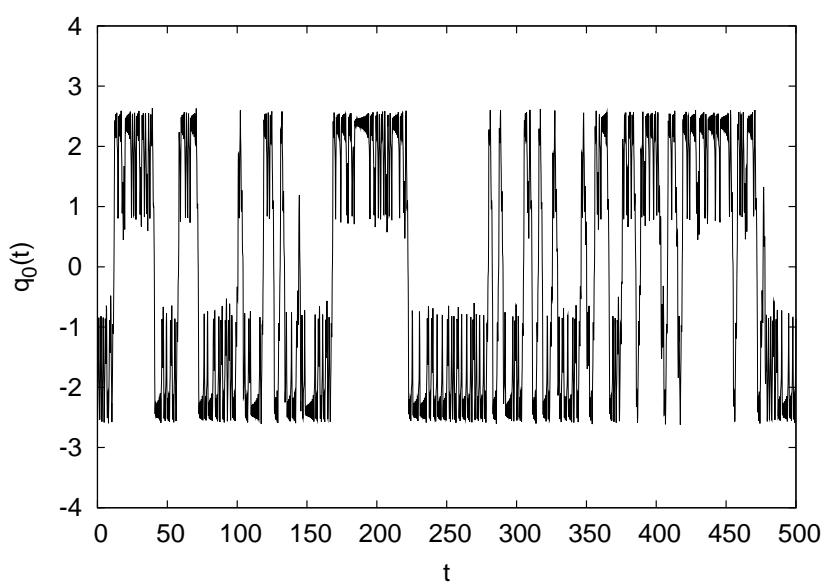

Fig. 9. DW model with $\tilde{\epsilon}=1$ : time signal sample of the slow variable $q_{0}(t)$. The ratio between fast and slow characteristic times is $\epsilon \sim 0.1$ (see text).

slow variable $x=q_{0}$ :

$\frac{d x}{d t}=-\frac{\partial V}{\partial x}=2 \sqrt{H} x-x^{3}$

with

$V(x)=H-\sqrt{H} x^{2}+\frac{1}{4} x^{4}$

The system (21) has one unstable steady state in $x_{0}=0$ corresponding to the top of the hill of height $H$, and two stable steady states in $x_{1 / 2}= \pm(4 H)^{1 / 4}$, i.e. the bottom of the valleys. The presence of the coupling $(c \neq 0)$ between slow and fast variables can induce transitions between the two valleys. The parameters in Eqs. (17), (18), (19), and (20) are fixed to the following values: $\sigma_{L}=10, r_{L}=28, b_{L}=8 / 3$, i.e. the classical set-up corresponding to the chaotic regime for the Lorenz-63 system; $H=4$, the height of the barrier; $c=0.5$, the coupling constant that rules the transition time scale of $q_{0}(t)$ between the two valleys; by setting $\tilde{\epsilon}=1$, the ratio $\epsilon$ between fast and slow characteristic times, see Eqs. (7) and (8), is $O\left(10^{-1}\right)$.

Since the time scale of the $q_{0}(t)$ well-to-well transitions may be considerably longer, depending on $1 / c$, than the characteristic time of $q_{1}(t)$, of order $O(1)$, we refer to $q_{0}$ as the slow variable, or the low-frequency observable, and to $q_{1}$ as the fast variable, or the high-frequency forcing, of the deterministic DW model. It can be easily shown that, for $c=0$, small perturbations $\Delta q_{0}$ around the two potential minima at $\pm(4 H)^{1 / 4}$ relax exponentially to zero with characteristic time $1 / 4 \sqrt{H}$. For sufficiently large values of $c$, the climatic variable $q_{0}(t)$ jumps aperiodically back and forth between the two valleys, driven by the chaotic signal $q_{1}(t)$, see Figs. 9 and 10.

The main statistical quantities investigated to analyse the DW model are the following:

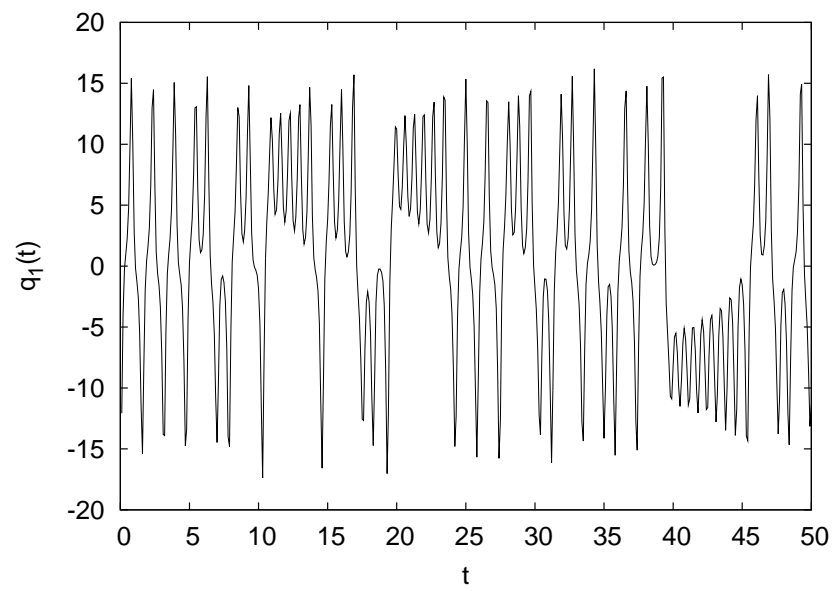

Fig. 10. DW model with $\tilde{\epsilon}=1$ : time signal sample of the fast variable $q_{1}(t)$.

a) the probability density function of the slow variable $q_{0}$;

b) the probability density function of the well-to-well transition time $t_{e}, \rho\left(t_{e}\right)$;

c) the slow and fast auto-correlation functions (ACF) $C_{i i}(t)=\left\langle q_{i}(t) q_{i}(0)\right\rangle /\left\langle q_{i}^{2}\right\rangle$, with $i=0,1 ;$

d) the slow and fast self-response functions (ARF) $R_{i i}(t)=\overline{\delta q_{i}(t) / \delta q_{i}(0)}$, with $i=0,1$;

e) the quadratic cross-response function of the slow variable $q_{0}(t)$ with respect to the fast variable $q_{1}(0)$.

Of course $R_{01}(t)$, i.e. the mean response of $q_{0}(t)$ to a perturbation on $q_{1}(0)$, is zero for trivial symmetry arguments. On the other hand, the quadratic response:

$R_{01}^{(q)}(t)=\frac{\left[\overline{\delta q_{0}(t)^{2}}\right]^{1 / 2}}{\delta q_{1}(0)}$

can give relevant physical information. Even in this case, since in all simulations the initial perturbation on $q_{1}(0)$ is kept constant, $\delta q_{1}(0)=\Delta \ll\left\langle q_{1}^{2}\right\rangle^{1 / 2}$, it is convenient to define as mean quadratic response of the slow variable $(s)$ with respect to the fast variable $(f)$ the quantity $R_{s f}^{(q)}(t)=\Delta \cdot R_{01}^{(q)}(t)$. The long-time saturation level of $R_{s f}^{(q)}(t)$ is of the order of the distance between the two climatic states.

With the current set-up, slow and fast variable have characteristic times which differ by an order of magnitude from each other, while the statistics of $q_{0}$ is strongly non Gaussian. Because of the skew structure of the system, i.e. the fast dynamics drives the slow dynamics but without counterfeedback, one expects that, at the least in the limit of large time scale separation, the joint PDF can be factorized, with an asymptotic PDF for $q_{0}$ of the form $\rho_{0}=K \cdot e^{-V_{\text {eff }}\left(q_{0}\right)}$, where $K$ is a normalization constant. 


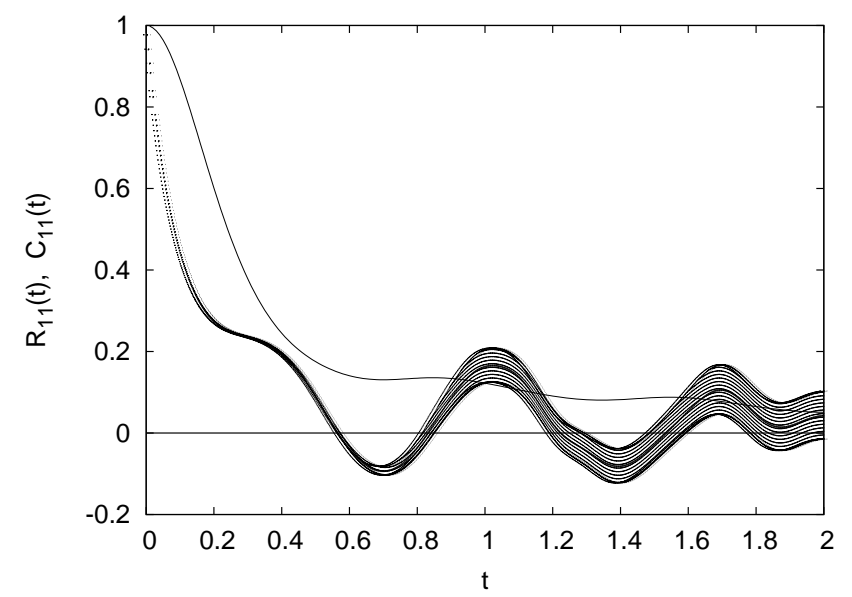

Fig. 11. DW model with $\tilde{\epsilon}=1$ : autocorrelation $C_{11}(t)$ (full line) and self-response $R_{11}(t)$, with statistical error bars, for the fast variable $q_{1}$.

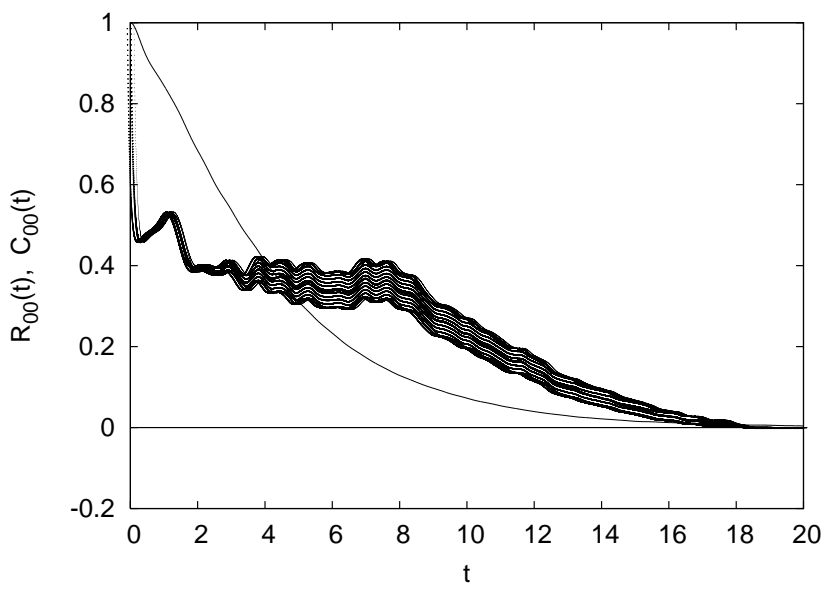

Fig. 12. DW model with $\tilde{\epsilon}=1$ : Autocorrelation $C_{00}(t)$ (full line) and self-response $R_{00}(t)$, with statistical error bars, for the slow variable $q_{0}$.

The FR properties of the deterministic DW model, for the fast and slow variables, are shown in Figs. 11 and 12, respectively.

The slow self-response $R_{00}(t)$ initially decreases exponentially with characteristic time $1 / 4 \sqrt{H}(H=4)$, i.e. the same behavior of the relaxation of a small perturbation near the bottom of a valley for $c=0$. Then, $R_{00}(t)$ relaxes to zero much more slowly. It is natural to assume that this is due to the long-time jumps between the valleys. It is well evident that $R_{00}$ behaves rather differently from $C_{00}$, while $R_{11}$ and $C_{11}$ have, at least, the same qualitative shape. On the other hand, the autocorrelation (self-response) time scales of the two variables differ from each other of a factor $\sim 10$, compatibly with the fact that the ratio between fast and slow characteristic times is $\epsilon \sim 0.1$, for the current set-up $(\widetilde{\epsilon}=1)$.

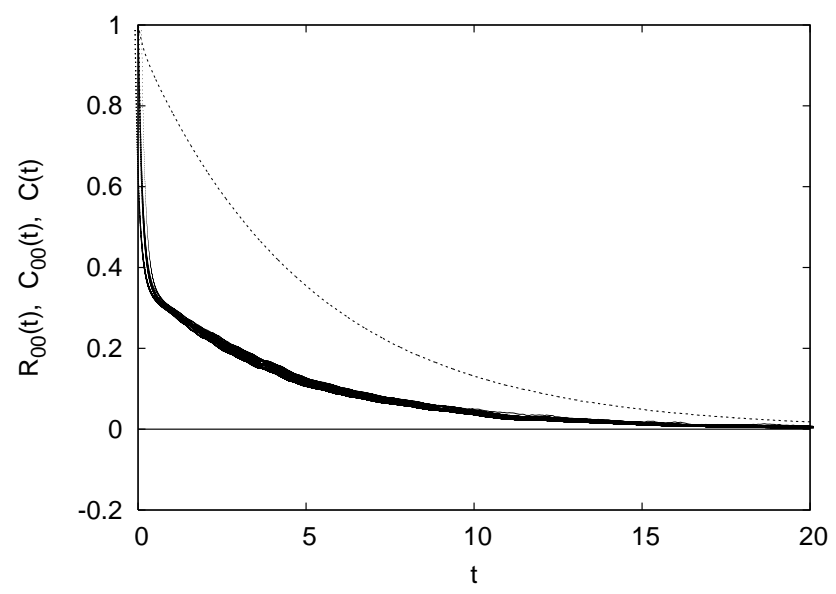

Fig. 13. DW model with $\widetilde{\epsilon}=0.01$, implying $\epsilon \sim 10^{-3}$ : autocorrelation $C_{00}(t)$ (dashed line), self-response $R_{00}(t)$, with statistical error bars, and the correlation function $C(t)$ predicted by the FRR (full line) which is actually undistinguishable from the response.

Since the statistics is far from being Gaussian, the "correct" correlation function which satisfies the FR theorem, for the slow variable, has the form:

$C(t)=-\left\langle\left. q_{0}(t) \frac{\partial \rho_{\epsilon}\left(q_{0}, q_{1}, q_{2}, q_{3}\right)}{\partial q_{0}}\right|_{t=0}\right\rangle$

where $\rho_{\epsilon}\left(q_{0}, q_{1}, q_{2}, q_{3}\right)$ is the (unknown) joint PDF of the state variable of the system at a fixed $\epsilon$. In the limit of large time separation, i.e. for $\widetilde{\epsilon} \rightarrow 0$, one expects that the asymptotic $\operatorname{PDF} \rho_{0}\left(q_{0}, q_{1}, q_{2}, q_{3}\right)$ is factorized:

$\rho_{0}\left(q_{0}, q_{1}, q_{2}, q_{3}\right)=K e^{-V_{\text {eff }}\left(q_{0}\right)} \rho_{L}\left(q_{1}, q_{2}, q_{3}\right)$

where $K$ is a normalization constant, and $\rho_{L}$ is the PDF of the Lorenz-63 state variable. Under this condition, the right correlation function predicted by the FRR has a relatively simple form:

$C(t)=\left\langle\left. q_{0}(t) \frac{\partial V_{\mathrm{eff}}\left(q_{0}\right)}{\partial q_{0}}\right|_{t=0}\right\rangle$

where $V_{\text {eff }}$ indicates the effective potential. For $\epsilon \sim 10^{-1}$ (corresponding to $\widetilde{\epsilon}=1$ ) we have checked numerically that the joint PDF is not yet factorized, while for a very small ratio between the characteristic times, $\epsilon \sim 10^{-3}$ (corresponding to $\widetilde{\epsilon}=10^{-2}$ ), the form (24) holds and, taking $V_{\text {eff }} \propto V$, we obtain a very good agreement between $R_{00}(t)$ and $C(t)$, see Fig. 13.

The cross-response properties of the DW model, measured by the quantity $R_{s f}^{(q)}(t)$, are reported in Fig. 18. We will consider again later this issue when discussing the stochastic modeling. While the mean (slow-to-fast) cross-response is null (not shown), its fluctuations grow with time. This means that an initial uncertainty on the fast variables has consequences for the predictability of the slow variable, since it 


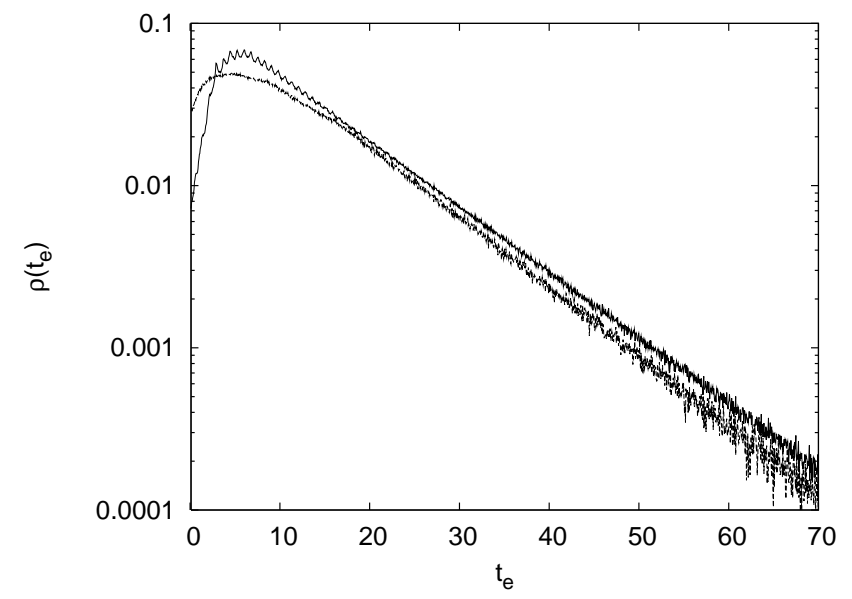

Fig. 14. Comparison of the PDFs of the transition time $t_{e}$ between the two climatic states for the DW model (full line) and the WNDW model (dashed line), for $\widetilde{\epsilon}=1(\epsilon \sim 0.1)$.

induces a mean separation growth between two initially close "climatic" states of the $q_{0}$ variable. At small times, $R_{s f}^{(q)}(t)$ grows exponentially in time, i.e. it is driven by the chaotic character of the fast variable while, at very long times, the well-to-well aperiodic jumps play the dominant role and the growth speed eventually decreases to zero until saturation sets in.

Let us now consider a stochastic model for the slow variable $q_{0}(t)$, obtained by replacing the fast variable $q_{1}$, in the equation for $q_{0}$, with a white noise. One has a Langevin equation of the kind:

$\frac{d q_{0}}{d t}(t)=2 \sqrt{H} q_{0}(t)-q_{0}^{3}+\sigma \cdot \xi(t)$

where $\xi(t)$ is a Gaussian process with $\langle\xi(t)\rangle=0$ and $\left\langle\xi(t) \xi\left(t^{\prime}\right)\right\rangle=\delta\left(t-t^{\prime}\right)$. We call Eq. (26) the WNDW model. The value $\sigma=19.75$ is determined by requiring that the PDFs of the well-to-well transition times have the same asymptotic behavior (i.e. exponential tail with the same exponent), see Fig. 14.

Let us notice that, in this case, because of the skew structure of the original system, the stochastic modeling is (relatively) simple and, differently from the generic case, the noise is additive. The time signal $q_{0}(t)$ obtained from the WNWD model is reported in Fig. 15. One observes strong similarities in the long-time transition statistics with respect to the deterministic model, even though the PDFs of the slow variable are quite different from one another, see Fig. 16.

The FR properties of the WNDW model are reported in Fig. 17. The slow variable is distributed according to $\sim e^{-V\left(q_{0}\right) / K}$, with $K=\sigma^{2} / 2$, and the FR theorem prediction is verified, i.e. one has a good agreement between $R_{00}(t)$ and the correlation function $C(t)$.

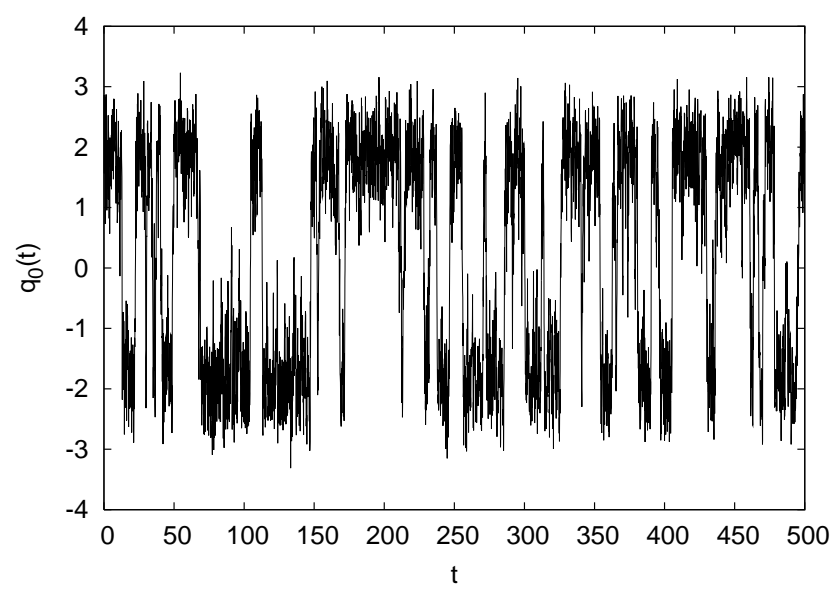

Fig. 15. WNDW model: time signal sample of the slow variable $q_{0}(t)$.

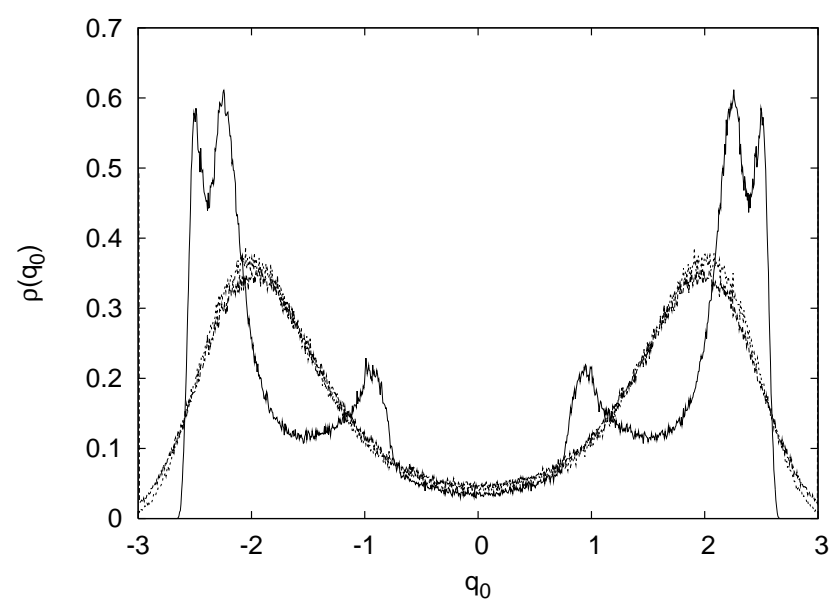

Fig. 16. PDFs of the slow variable $q_{0}$ for the DW model with $\tilde{\epsilon}=1$, i.e. $\epsilon \sim 0.1$ (full line), the WNDW model (dashed line) and the DW model with $\tilde{\epsilon}=10^{-2}$, i.e. $\epsilon \sim 10^{-3}$ (dotted line). In the limit $\epsilon \rightarrow 0$, the PDFs of the deterministic model and of the stochastic model collapse.

We redefine, as already seen when discussing the stochastic model approximating the Lorenz-96 system, the quadratic cross-response function $R_{s f}^{(q)}(t)$ as the root mean square growth of the error $\delta q_{0}(t)$ induced by two different noise realizations.

In Fig. 18, the behavior of $R_{s f}^{(q)}(t)$ for the deterministic DW system and its stochastic model is reported. The WNDW model is not able to reproduce the two-time behavior of the deterministic model, mainly due to the impossibility to control the amplitude of the initial perturbation. Because of that, the error on the climatic state of the system saturate very quickly, as soon as the trajectory starts jumping between the wells. 


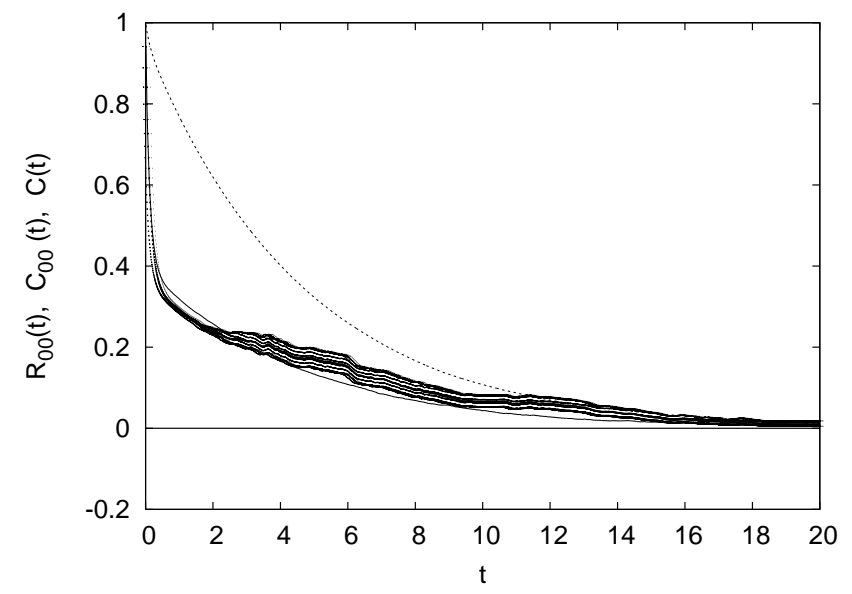

Fig. 17. WNDW model: autocorrelation $C_{00}(t)$ (dashed line), selfresponse $R_{00}(t)$, with statistical error bars, and the correlation function $C(t)$ predicted by the FRR (full line).

\section{Discussion and conclusive remarks}

In this paper we have presented a detailed investigation of the Fluctuation-Response properties of chaotic systems with fast and slow dynamics. The numerical study has been performed on two models, namely the 360-variable Lorenz-96 system, with reciprocal feedback between fast and slow variables, and a simplified low dimensional system, both of which are able to capture the main features, and related difficulties, typical of the multiscale systems. The first point we wish to emphasize is how, even in non Hamiltonian systems, a generalized Fluctuation-Response Relation (FRR) holds. This allows for a link between the average relaxation of perturbations and the statistical properties (correlation functions) of the unperturbed system. Although one has non Gaussian statistics, the correlation functions of the slow (fast) variables have at least a qualitative resemblance with the response functions to perturbations on the slow (fast) degrees of freedom. The average response function of a slow variable to perturbations of the fast degrees of freedom is zero, nevertheless the impact of the fast dynamics on the slowly varying components cannot be neglected. This fact is clearly highlighted by the behavior of a suitable quadratic response function. Such a phenomenon, which can be regarded as a sort of sensitivity of the slow variables to variations of the fast components, has an important consequence for the modeling of the slow dynamics in terms of a Langevin equation. Even an optimal model (i.e. able to mimic autocorrelation and selfresponse of the slow variable), beyond a certain intrinsic time interval, can give just statistical predictions, in the sense that, at most, one can hope to have an agreement among the statistical features of system and model. In stochastic dynamical systems, one has to deal with a similar behavior: the relevant "complexity" of the systems is obtained by considering the

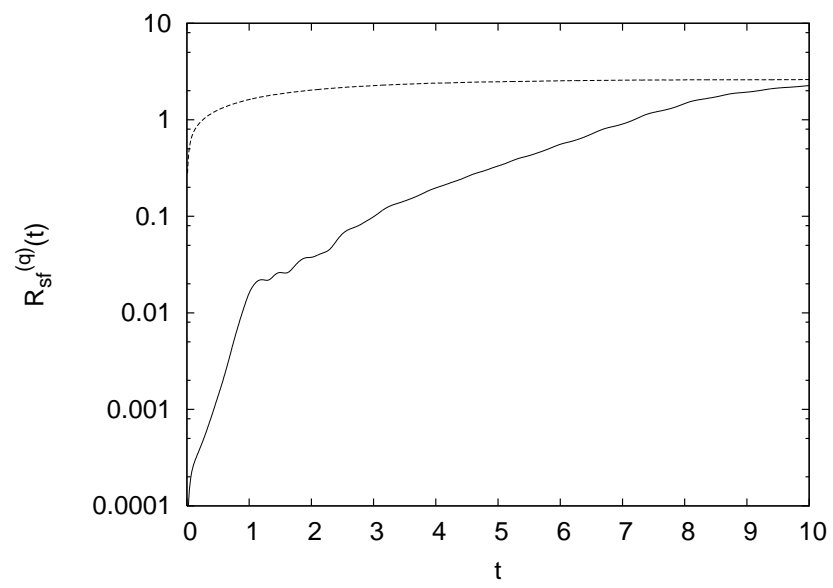

Fig. 18. Quadratic cross-response function $R_{s f}^{(q)}(t)$ for the DW model (full line) and the WNDW model (dashed line). The growth rates of $R_{s f}^{(q)}(t)$ for the DW model are compatible with the two characteristic times of the system, while for the WNDW model $R_{s f}^{(q)}(t)$ quickly saturates in a very short time.

divergence of nearby trajectories evolving under two different noise realizations. Therefore a good model for the slow dynamics (e.g. a Langevin equation) must show a sensitivity to the noise.

\section{Appendix A}

\section{Generalized FRR}

In this Appendix we give a derivation, under general rather hypothesis, of a generalized FRR. Consider a dynamical system $\mathbf{x}(0) \rightarrow \mathbf{x}(t)=U^{t} \mathbf{x}(0)$ with states $\mathbf{x}$ belonging to a $N$ dimensional vector space. For the sake of generality, we will consider the case in which the time evolution can also be not completely deterministic (e.g. stochastic differential equations). We assume the existence of an invariant probability distribution $\rho(\mathbf{x})$, for which some "absolute continuity" type conditions are required (see later), and the mixing character of the system (from which its ergodicity follows). Note that no assumption is made on $N$.

Our aim is to express the average response of a generic observable $A$ to a perturbation, in terms of suitable correlation functions, computed according to the invariant measure of the unperturbed system. At the first step we study the behavior of one component of $\mathbf{x}$, say $x_{i}$, when the system, described by $\rho(\mathbf{x})$, is subjected to an initial (non-random) perturbation such that $\mathbf{x}(0) \rightarrow \mathbf{x}(0)+\Delta \mathbf{x}_{0}$. This instantaneous kick $^{3}$ modifies the density of the system into $\rho^{\prime}(\mathbf{x})$, related to

\footnotetext{
${ }^{3}$ The study of an "impulsive" perturbation is not a severe limitation, e.g. in the linear regime from the (differential) linear response
} 
the invariant distribution by $\rho^{\prime}(\mathbf{x})=\rho\left(\mathbf{x}-\Delta \mathbf{x}_{0}\right)$. We introduce the probability of transition from $\mathbf{x}_{0}$ at time 0 to $\mathbf{x}$ at time $t, W\left(\mathbf{x}_{0}, 0 \rightarrow \mathbf{x}, t\right)$. For a deterministic system, with evolution law $\mathbf{x}(t)=U^{t} \mathbf{x}(0)$, the probability of transition reduces to $W\left(\mathbf{x}_{0}, 0 \rightarrow \mathbf{x}, t\right)=\delta\left(\mathbf{x}-U^{t} \mathbf{x}_{0}\right)$, where $\delta(\cdot)$ is the Dirac's delta. Then we can write an expression for the mean value of the variable $x_{i}$, computed with the density of the perturbed system:

$\left\langle x_{i}(t)\right\rangle^{\prime}=\iint x_{i} \rho^{\prime}\left(\mathbf{x}_{0}\right) W\left(\mathbf{x}_{0}, 0 \rightarrow \mathbf{x}, t\right) d \mathbf{x} d \mathbf{x}_{0}$.

The mean value of $x_{i}$ during the unperturbed evolution can be written in a similar way:

$\left\langle x_{i}(t)\right\rangle=\iint x_{i} \rho\left(\mathbf{x}_{0}\right) W\left(\mathbf{x}_{0}, 0 \rightarrow \mathbf{x}, t\right) d \mathbf{x} d \mathbf{x}_{0}$.

Therefore, defining $\overline{\delta x_{i}}=\left\langle x_{i}\right\rangle^{\prime}-\left\langle x_{i}\right\rangle$, we have:

$$
\begin{aligned}
\overline{\delta x_{i}}(t) & =\iint x_{i} F\left(\mathbf{x}_{0}, \Delta \mathbf{x}_{0}\right) \rho\left(\mathbf{x}_{0}\right) W\left(\mathbf{x}_{\mathbf{0}}, 0 \rightarrow \mathbf{x}, t\right) d \mathbf{x} d \mathbf{x}_{0} \\
& =\left\langle x_{i}(t) F\left(\mathbf{x}_{0}, \Delta \mathbf{x}_{0}\right)\right\rangle
\end{aligned}
$$

where

$F\left(\mathbf{x}_{0}, \Delta \mathbf{x}_{0}\right)=\left[\frac{\rho\left(\mathbf{x}_{0}-\Delta \mathbf{x}_{0}\right)-\rho\left(\mathbf{x}_{0}\right)}{\rho\left(\mathbf{x}_{0}\right)}\right]$.

Let us note here that the mixing property of the system is required so that the decay to zero of the time-correlation functions assures the switching off of the deviations from equilibrium.

For an infinitesimal perturbation $\delta \mathbf{x}(0)=\left(\delta x_{1}(0) \cdots \delta x_{N}\right.$ $(0))$, if $\rho(\mathbf{x})$ is non-vanishing and differentiable, the function in Eq. (A4) can be expanded to first order and one obtains:

$$
\begin{aligned}
\overline{\delta x_{i}}(t) & =-\sum_{j}\left\langle\left. x_{i}(t) \frac{\partial \ln \rho(\mathbf{x})}{\partial x_{j}}\right|_{t=0}\right\rangle \delta x_{j}(0) \\
& \equiv \sum_{j} R_{i j}(t) \delta x_{j}(0)
\end{aligned}
$$

which defines the linear response

$$
R_{i j}(t)=-\left\langle\left. x_{i}(t) \frac{\partial \ln \rho(\mathbf{x})}{\partial x_{j}}\right|_{t=0}\right\rangle
$$

of the variable $x_{i}$ with respect to a perturbation of $x_{j}$. One can easily repeat the computation for a generic observable $A(\mathbf{x})$ :

$\overline{\delta A}(t)=-\sum_{j}\left\langle\left. A(\mathbf{x}(t)) \frac{\partial \ln \rho(\mathbf{x})}{\partial x_{j}}\right|_{t=0}\right\rangle \delta x_{j}(0)$.

For Langevin equations, the differentiability of $\rho(\boldsymbol{X})$ is well established. On the contrary, one could argue that in a one understands the effect of a generic perturbation. chaotic deterministic dissipative system the above machinery cannot be applied, because the invariant measure is not smooth at all. Typically the invariant measure of a chaotic attractor has a multifractal character and its Renyi dimensions $d_{q}$ are not constant (Paladin and Vulpiani, 1987). In chaotic dissipative systems the invariant measure is singular, however the previous derivation of the FRR is still valid if one considers perturbations along the expanding directions. For a mathematically oriented presentation see Ruelle (1998). A general response function has two contributions, corresponding respectively to the expanding (unstable) and the contracting (stable) directions of the dynamics. The first contribution can be associated to some correlation function of the dynamics on the attractor (i.e. the unperturbed system). On the contrary this is not true for the second contribution (from the contracting directions), this part to the response is very difficult to extract numerically (Cessac and Sepulchre, 2007). In chaotic deterministic systems, in order to have a differentiable invariant measure, one has to invoke the stochastic regularization (Zeeman, 1990). If such a method is not feasible, one can use the direct approach by Abramov and Majda (2007). For a study of the FRR in chaotic atmospheric systems, see Dymnikov and Gritsoun (2005) and Gritsoun and Branstator (2007).

Let us notice that a small amount of noise, that is always present in a physical system, smoothen the $\rho(\mathbf{x})$ and the FRR can be derived. We recall that this "beneficial" noise has the important role of selecting the natural measure, and, in the numerical experiments, it is provided by the round-off errors of the computer. We stress that the assumption on the smoothness of the invariant measure allows to avoid subtle technical difficulties.

\section{Appendix B}

\section{A general remark on the decay of correlation functions}

Using some general arguments one has that all the (typical) correlation functions at large time delay have to relax to zero with the same characteristic time, related to spectral properties of the operator $\hat{\mathbf{L}}$ which rules the time evolution of the $P(X, t)$ :

$\frac{\partial}{\partial t} P(\boldsymbol{X}, t)=\hat{\mathbf{L}} P(\boldsymbol{X}, t)$.

In the case of ordinary differential equations

$d X_{i} / d t=Q_{i}(\boldsymbol{X}) \quad i=1, \cdots, N$

the operator $\hat{\mathbf{L}}$ has the shape

$\hat{\mathbf{L}} P(\boldsymbol{X}, t)=-\sum_{i} \frac{\partial}{\partial X_{i}}\left(Q_{i}(\boldsymbol{X}) P(\boldsymbol{X}, t)\right)$. 
For Langevin equations i.e. in Eq. (B2) $Q_{i}$ is replaced by $Q_{i}+\eta_{i}$ where $\left\{\eta_{i}\right\}$ are Gaussian processes with $<\eta_{i}(t)>=0$ and $<\eta_{i}(t) \eta_{j}\left(t^{\prime}\right)>=2 \Lambda_{i, j} \delta\left(t-t^{\prime}\right)$, one has

$$
\begin{aligned}
\hat{\mathbf{L}} P(\boldsymbol{X}, t)= & -\sum_{i} \frac{\partial}{\partial X_{i}}\left(Q_{i}(\boldsymbol{X}) P(\boldsymbol{X}, t)\right) \\
& +\sum_{a b} \Lambda_{i, j} \frac{\partial^{2}}{\partial X_{i} \partial X_{i}} P(\boldsymbol{X}, t) .
\end{aligned}
$$

Let us introduce the eigenvalues $\left\{\alpha_{k}\right\}$ and the eigenfunctions $\left\{\psi_{k}\right\}$ of $\mathcal{L}$ :

$\hat{L} \psi_{k}=\alpha_{k} \psi_{k}$.

Of course $\psi_{0}=P_{i n v}$ and $\alpha_{0}=0$, and typically in mixing systems Re $\alpha_{k}<0$ for $k=1,2, \ldots$. Furthermore assuming that coefficient $\left\{g_{1}, g_{2}, \ldots\right\}$ and $\left\{h_{1}, h_{2}, \ldots\right\}$ exist such that functions $g(\boldsymbol{X})$ and $h(\boldsymbol{X})$ are uniquely expanded as

$g(\boldsymbol{X})=\sum_{k=0} g_{k} \psi_{k}(\boldsymbol{X}), h(\boldsymbol{X})=\sum_{k=0} h_{k} \psi_{k}(\boldsymbol{X})$,

so we have

$C_{g, f}(t)=\sum_{k=1} g_{k} h_{k}<\psi_{k}^{2}>e^{\alpha_{k} t}$,

where $\quad C_{g, f}(t)=<g(\boldsymbol{X}(t)) h(\boldsymbol{X}(t))>-<g(\boldsymbol{X})><h(\boldsymbol{X})>$. For "generic" functions $g$ and $f$, i.e. if they are not orthogonal to $\psi_{1}$ so that $g_{1} \neq 0$ and $h_{1} \neq 0$, at large time the correlation $C_{g, f}(t)$ approaches to zero as

$C_{g, f}(t) \sim e^{-t / \tau_{c}}, \quad \tau_{c}=\frac{1}{\left|\operatorname{Re} \alpha_{1}\right|}$.

In some cases, e.g. very intermittent systems like the Lorenz model at $r \simeq 166.07, \operatorname{Re} \alpha_{1}=0$ so the decay is not exponentially fast.

\section{Appendix C}

\section{Lyapunov exponent in dynamical systems with noise}

In systems with noise, the simplest way to introduce the Lyapunov exponent is to treat the random term as a timedependent term. Basically one considers the separation of two close trajectories with the same realization of noise. Only for sake of simplicity consider a one-dimensional Langevin equation

$$
\frac{d x}{d t}=-\frac{\partial V(x)}{\partial x}+\sigma \eta,
$$

where $\eta(t)$ is a white noise and $V(x)$ diverges for $|x| \rightarrow \infty$, like, e.g., the usual double well potential $V=-x^{2} / 2+x^{4} / 4$.

The Lyapunov exponent $\lambda_{\sigma}$, associated with the separation rate of two nearby trajectories with the same realization of $\eta(t)$, is defined as

$\lambda_{\sigma}=\lim _{t \rightarrow \infty} \frac{1}{t} \ln |z(t)|$ where the evolution of the tangent vector is given by:

$$
\frac{d z}{d t}=-\frac{\partial^{2} V(x(t))}{\partial x^{2}} z(t) .
$$

The quantity $\lambda_{\sigma}$ obtained in the previous way, although well defined, i.e. the Oseledec theorem (Bohr et al., 1998) holds, it is not always a useful characterization of complexity.

Since the system is ergodic with invariant probability distribution $P(x)=C_{1} e^{-V(x) / C_{2}}$, where $C_{1}$ is a normalization constant and $C_{2}=\sigma^{2} / 2$, one has:

$$
\begin{aligned}
\lambda_{\sigma} & =\lim _{t \rightarrow \infty} \frac{1}{t} \ln |z(t)| \\
& =-\lim _{t \rightarrow \infty} \frac{1}{t} \int_{0}^{t} \partial_{x x}^{2} V\left(x\left(t^{\prime}\right)\right) d t^{\prime} \\
& =-C_{1} \int \partial_{x x}^{2} V(x) e^{-V(x) / C_{2}} d x \\
& =-\frac{C_{1}}{C_{2}} \int\left(\partial_{x} V(x)\right)^{2} e^{-V(x) / C_{2}} d x<0 .
\end{aligned}
$$

This has a rather intuitive meaning: the trajectory $x(t)$ spends most of the time in one of the "valleys" where $-\partial_{x x}^{2} V(x)<0$ and only short intervals on the "hills" where $-\partial_{x x}^{2} V(x)>0$, so that the distance between two trajectories evolving with the same noise realization decreases on average. The previous result for the 1-D Langevin equation can easily be generalized to any dimension for gradient systems if the noise is small enough (Loreto et al., 1996).

A negative value of $\lambda_{\sigma}$ implies a fully predictable process only if the realization of the noise is known. In the case of two initially close trajectories evolving under two different noise realizations, after a certain time $T_{\sigma}$, the two trajectories can be very distant, because they can be in two different valleys. For $\sigma \rightarrow 0$, due to the Kramers formula (Gardiner, 1990), one has $T_{\sigma} \sim e^{\Delta V / \sigma^{2}}$, where $\Delta V$ is the difference between the values of $V$ on the top of the hill and at the bottom of the valley.

Let us now discuss the main difficulties in defining the notion of "complexity" of an evolution law with a random perturbation, discussing a simple case. Consider the 1-D map

$x(t+1)=f[x(t), t]+\sigma w(t)$,

where $t$ is an integer and $w(t)$ is an uncorrelated random process, e.g. $w$ are independent random variables uniformly distributed in $[-1 / 2,1 / 2]$. For the largest LE $\lambda_{\sigma}$, as defined in (C2), now one has to study the equation

$z(t+1)=f^{\prime}[x(t), t] z(t)$,

where $f^{\prime}=d f / d x$.

Following the approach in (Paladin et al., 1995) let $x(t)$ be the trajectory starting at $x(0)$ and $x^{\prime}(t)$ be the trajectory starting from $x^{\prime}(0)=x(0)+\delta x(0)$. Let $\delta_{0} \equiv|\delta x(0)|$ and indicate by $\tau_{1}$ the minimum time such that $\left|x^{\prime}\left(\tau_{1}\right)-x\left(\tau_{1}\right)\right| \geq \Delta$. Then, we put $x^{\prime}\left(\tau_{1}\right)=x\left(\tau_{1}\right)+\delta x(0)$ and define $\tau_{2}$ as the time such that $\left|x^{\prime}\left(\tau_{1}+\tau_{2}\right)-x\left(\tau_{1}+\tau_{2}\right)\right|>\Delta$ for the first time, and so on. In this way the Lyapunov exponent can be defined as

$\lambda=\frac{1}{\bar{\tau}} \ln \left(\frac{\Delta}{\delta_{0}}\right)$ 
being $\bar{\tau}=\sum \tau_{i} / \mathcal{N}$ where $\mathcal{N}$ is the number of the intervals in the sequence. If the above procedure is applied by considering the same noise realization for both trajectories, $\lambda$ in Eq. (C2) coincides with $\lambda_{\sigma}$ (if $\lambda_{\sigma}>0$ ). Differently, by considering two different realizations of the noise for the two trajectories, we have a new quantity

$$
K_{\sigma}=\frac{1}{\bar{\tau}} \ln \left(\frac{\Delta}{\delta_{0}}\right)
$$

which naturally arises in the framework of information theory and algorithmic complexity theory: note that $K_{\sigma} / \ln 2$ is the number of bits per unit time one has to specify in order to transmit the sequence with a precision $\delta_{0}$, The generalization of the above treatment to $N$-dimensional maps or to ordinary differential equations is straightforward.

If the fluctuations of the effective Lyapunov exponent $\gamma(t)$ (in the case of Eq. C5 $\gamma(t)$ is nothing but $\left.\ln \left|f^{\prime}(x(t))\right|\right)$ are very small (i.e. weak intermittency) one has $K_{\sigma}=\lambda+O(\sigma / \Delta)$.

The interesting situation happens for strong intermittency when there are alternations of positive and negative $\gamma$ during long time intervals: this induces a dramatic change for the value of $K_{\sigma}$. Numerical results on intermittent maps (Paladin et al., 1995) show that the same system can be regarded either as regular (i.e. $\lambda_{\sigma}<0$ ), when the same noise realization is considered for two nearby trajectories, or as chaotic (i.e. $K_{\sigma}>0$ ), when two different noise realizations are considered. We can say that a negative $\lambda_{\sigma}$ for some value of $\sigma$ in not an indication that "noise induces order"; a correct conclusion is that noise can induce synchronization.

Acknowledgements. We warmly thank A. Mazzino, S. Musacchio, R. Pasmanter and A. Puglisi for interesting discussions and suggestions, and two anonymous referees for their contructive criticism in reviewing this paper.

Edited by: O. Talagrand

Reviewed by: two anonymous referees

\section{References}

Abramov, R. and Majda, A.: New approximations and tests of linear fluctuation-response for chaotic nonlinear forced-dissipative dynamical systems, J. Nonlinear Sci., accepted, 2007.

Bell, T. L.: Climate sensitivity from fluctuation-dissipation: some simple model tests, J. Atmos. Sci., 37, 1700-1707, 1980.

Biferale, L., Daumont, I., Lacorata, G., and Vulpiani, A.: Fluctuation-response relation in turbulent systems, Phys. Rev. E, 65, 016302, 016302-1-016302-7, 2002.

Boffetta, G., Lacorata, G., Musacchio, S., and Vulpiani, A.: Relaxation of finite perturbations: Beyond the Fluctuation-Response relation, Chaos, 13, 806-811, 2003.

Bohr, T., Jensen, M. H., Paladin, G., and Vulpiani, A.: Dynamical systems approach to turbulence, Cambridge University Press, UK, 1998.
Cane, M.: Understanding and predicting the world's climate system, Chaos in geophysical flows, Chaos in geophysical flows, edited by: Boffetta, G., Lacorata, G., Visconti, G., and Vulpiani, A., Otto Eds., Torino, Italy, 2003.

Cessac, B. and Sepulchre, J.-A.: Linear response, susceptibility and resonance in chaotic toy models, Physica D, 225, 13-28, 2007.

Cionni, I., Visconti, G., and Sassi, F.: Fluctuation-dissipation theorem in a general circulation model, Geophys. Res. Lett., 31, L09206, doi:10.1029/2004GL019739, 2004.

Deker, U. and Haake, F.: Fluctuation-dissipation theorems for classical processes, Phys. Rev. A, 11, 2043-2056, 1975.

Ditlevsen, P. D.: Observation of $\alpha$-stable noise induced millennial climate changes from an ice-core record, Geophys. Res. Lett., 26(10), 1441-1444, 1999.

Dymnikov, V. P.: Potential Predictability of Large-Scale Atmospheric Processes, Izvestiya, Atmospheric and Oceanic Physics, 40(5), 513-519, 2004.

Dymnikov, V. P. and Gritsoun, A. S.: Climate model attractors: chaos, quasi-regularity and sensitivity to small perturbations of external forcing, Nonlin. Processes Geophys., 8, 201-209, 2001, http://www.nonlin-processes-geophys.net/8/201/2001/.

Dymnikov, V. P. and Gritsoun, A. S.: Current problems in the mathematical theory of climate, Izvestiya, Atmospheric and Oceanic Physics, 41, 294-314, 2005.

Falcioni, M., Isola, S., and Vulpiani, A.: Correlation functions and relaxation properties in chaotic dynamics and statistical mechanics, Phys. Lett. A, 144, 341-346, 1990.

Fraedrich, K.: Predictability: short and long-term memory of the atmosphere, Chaos in geophysical flows, Chaos in geophysical flows, edited by: Boffetta, G., Lacorata, G., Visconti, G., and Vulpiani, A., Otto Eds., Torino, Italy, 2003.

Frisch, U.: Turbulence, Cambridge University Press, UK, 1995.

Gardiner, C. W.: Handbook of stochastic methods for Physics, Chemistry and the Natural Sciences, Springer-Verlag, Berlin, Germany, 1990.

Givon, D., Kupferman, R., and Stuart, A.: Extracting macroscopic dynamics: model problems and algorithms, Nonlinearity, 17, 55127, 2004.

Gritsoun, A. S.: Fluctuation-dissipation theorem on attractors of atmospheric models, Russ. J. Numer. Analysis Math. Modelling, 16, 115-133, 2001.

Gritsoun, A. S. and Branstator, G.: Climate Response Using a Three-Dimensional Operator Based on the Fluctuation Dissipation Theorem, J. Atmos. Sci., 64(7), 2558-2575, 2007.

Gritsoun, A. S., Branstator, G., and Dymnikov, V. P.: Construction of the linear response operator of an atmospheric general circulation model to small external forcing, Russ. J. Numer. Analysis Math. Modelling, 17, 399-416, 2002.

Gritsoun, A. S. and Dymnikov, V. P.: Barotropic atmosphere response to small external action: theory and numerical experiments, Izvestiya, Atmospheric and Oceanic Physics, 35, 511$525,1999$.

Haskins, R., Goody, R., and Chen, L.: Radiance covariance and climate models, J. Climate, 12, 1409-1422, 1999.

Hohenberg, P. C. and Shraiman, B. I.: Chaotic behavior of an extended system, Physica D, 37, 109-115, 1989.

Imkeller, P. and von Storch, J.-S. (Eds.): Stochastic Climate Models, Birkhäuser, Basel, Switzerland, 2001.

Kraichnan, R. H.: Classical fluctuation-relaxation theorem, Phys. 
Rev., 113, 1181-1182, 1959.

Kraichnan, R. H.: Deviations from fluctuation-relaxation relations, Physica A, 279, 30-36, 2000.

Kraichnan, R. H. and Montgomery, D.: Two-dimensional turbulence, Rep. Prog. Phys., 43, 547-619, 1980.

Kubo, R.: The fluctuation-dissipation theorem, Rep. Prog. Phys., 29, 255-284, 1966.

Kubo, R.: Brownian motion and nonequilibrium statistical mechanics, Science, 233, 330-334, 1986.

Leith, C. E.: Climate response and fluctuation-dissipation, J. Atmos. Sci., 32, 2022-2026, 1975.

Leith, C. E.: Predictability of climate, Nature, 276, 352-355, 1978.

Lorenz, E. N.: Deterministic nonperiodic flow, J. Atmos. Sci., 20, 130-141, 1963.

Lorenz, E. N.: Predictability - a problem partly solved, Proceedings, Seminar on Predictability ECMWF, 1, 1-18, 1996.

Loreto, V., Paladin, G., and Vulpiani, A.: On the concept of complexity for random dynamical systems, Phys. Rev. E, 53, $2087-$ 2098, 1996.

Majda, A. J., Abramov, R., and Grote, M.: Information theory and stochastics for multiscale nonlinear systems, CRM monograph series 25, American Mathematical Society, 2005.

Majda, A. J. and Franzke, C.: Low Order Stochastic Mode Reduction for a Prototype Atmospheric GCM, J. Atmos. Sci., 63(2), 457-479, 2006.

Majda, A. J., Timofeyev, I., and Vanden-Eijnden, E.: Models for stochastic climate prediction, Proc. Natl. Acad. Sci. USA, 96, 14687-14 691, 1999.

Majda, A. J., Timofeyev, I., and Vanden-Eijnden, E.: A mathematical framework for stochastic climate models, Commun. Pure Appl. Math., 54, 891-974, 2001.

Majda, A. J. and Wang, X.: Nonlinear Dynamics and Statistical Theories for Basic Geophysical Flows, Cambridge University Press, UK, 2006.

Marwan, N., Trauth, M., Vuille, M., and Kurths, J.: Comparing modern and Pleistocene ENSO-like influences in NW Argentina using nonlinear time series analysis methods, Clim. Dynam., 21, 317-326, 2003.
Matsumoto, K. and Tsuda, I.: Noise-induced order, J. Stat. Phys., 31, 87-106, 1983.

Mazzino, A., Musacchio, S., and Vulpiani, A.: Multiple scale analysis and renormalization for preasymptotic scalar transport, Phys. Rev. E, 71, 011113, 1-11, 2005.

McComb, W. D. and Kiyani, K.: Eulerian spectral closures for isotropic turbulence using a time-ordered fluctuation-dissipation relation, Phys. Rev. E, 72, 016309, 016309-1-016309-12, 2005.

Moeng, C.-H.: A Large-Eddy Simulation model for the study of planetary boundary layer turbulence, J. Atmos. Sci., 41, 20522062, 1984.

Moeng, C.-H. and Sullivan, P. P.: A comparison of shear and buoyancy driven planetary boundary layer flows, J. Atmos. Sci., 51, 999-1021, 1994.

North, G. R., Bell, R. E., and Hardin, J. W.: Fluctuation dissipation in a general circulation model, Clim. Dynam., 8, 259-264, 1993.

Paladin, G., Serva, M., and Vulpiani, A.: Complexity in dynamical systems with noise, Phys. Rev. Lett., 74, 66-69, 1995.

Paladin, G. and Vulpiani, A.: Anomalous scaling laws in multifractal objects, Phys. Rep., 156, 147-225, 1987.

Pasmanter, R. A.: On long-lived vortices in 2-D viscous flows, most probable states of inviscid 2-D flows and a soliton equation, Phys. Fluids, 6, 1236-1241, 1994.

Pikovsky, A., Rosenblum, M., and Kurths, J.: Synchronization: a universal concept in nonlinear sciences, Cambridge University Press, UK, 2003.

Rose, R. H. and Sulem, P. L.: Fully developed turbulence and statistical mechanics, J. Phys., 39, 441-484, 1978.

Ruelle, D.: General linear response formula in statistical mechanics, and the fluctuation-dissipation theorem far from equilibrium, Phys. Lett. A, 245, 220-224, 1998.

Sullivan, P. P., McWilliams, J.-C., and Moeng, C.-H.: A subgridscale model for large-eddy simulation of planetary boundary layer flows, Bound. Layer Meteorol., 71, 247-276, 1994.

Zeeman, E. C.: Stability of dynamical systems, Nonlinearity, 1, 115-135, 1988. 